\title{
Fatty acid-based polyurethane films for wound dressing applications
}

\author{
Guncem Gultekin • Cigdem Atalay-Oral • \\ Sibel Erkal · Fikret Sahin · Djursun Karastova • \\ S. Birgul Tantekin-Ersolmaz $\cdot$ F. Seniha Guner
}

Received: 22 May 2008/Accepted: 18 August 2008/Published online: 7 October 2008

(c) The Author(s) 2008. This article is published with open access at Springerlink.com

\begin{abstract}
Fatty acid-based polyurethane films were prepared for use as potential wound dressing material. The polymerization reaction was carried out with or without catalyst. Polymer films were prepared by casting-evaporation technique with or without crosslink-catalyst. The film prepared from uncatalyzed reaction product with crosslink-catalyst gave slightly higher crosslink density. The mechanical tests showed that, the increase in the tensile strength and decrease in the elongation at break is due to the increase in the degree of crosslinking. All films were flexible, and resisted to acid solution. The films prepared without crosslink-catalyst were more hydrophilic, absorbed more water. The highest permeability values were generally obtained for the films prepared without crosslink catalyst. Both the direct contact method and the MMT test were applied for determination of cytotoxicity of polymer films and the polyurethane film prepared from uncatalyzed reaction product without crosslink-catalyst showed better
\end{abstract}

G. Gultekin

Delft University of Technology, Faculty of Applied Sciences, Delft Chem Tech, P.O. Box 5045, 2600 GA Delft,

The Netherland

C. Atalay-Oral · S. B. Tantekin-Ersolmaz · F. S. Guner ( $₫)$

Chemical Engineering Department, Istanbul Technical

University, Maslak, Istanbul 34469, Turkey

e-mail: guners@itu.edu.tr

\section{S. Erkal}

Cebeci School of Health, University of Ankara, Altindag,

Ankara, Turkey

F. Sahin · D. Karastova

Faculty of Medicine, Microbiology and Clinical Microbiology,

University of Ankara, Ankara 06100, Turkey biocompatibility property, closest to the commercial product, Opsite ${ }^{\circledR}$.

\section{Introduction}

One of the most important renewable sources frequently used in polymer synthesis is triglyceride oils. They are not volatile, hence they do not develop volatile organic compounds (VCO). They are free of heavy metals, pesticides ect., hence they are green and clean, and they are fully biodegradable.

In order to improve end-product properties, triglyceride oils have been used in paint formulation since the 19th century. In particular, synthesis of polymers from triglyceride oils has become more popular after the petroleum shortage. Triglyceride oil-based polymers have many advantages compared to polymers prepared from petroleum-based monomers. They are more biodegradable and, in many cases, cheaper than petroleum-based polymers [1].

A triglyceride is an ester product obtained from one molecule of glycerol and three molecules of fatty acid [2]. The fatty acids contribute to $94-96 \%$ of the total weight of one molecule of triglyceride oil. Most of the triglyceride oil consists of different types of fatty acids. Depending on the fatty acid distribution, each type of oil has specific physical and chemical properties affecting the final properties of the prepared polymer.

Polymeric materials are widely used in biomedical applications due to their desired properties. Among these, polyurethanes are one of the most important classes of polymers due to their segmented (hard and soft) polymeric character obtained through the use of a chain extender which mainly determines their mechanical and physical 
properties $[3,4]$. In the last decade nonsegmented polyurethanes [5] which is usually the case when natural hydroxyl sources are used, have been synthesis for different purposes [6-9]. Polyurethanes are also recognized with their biocompatibility and biodegradation character [10] and hence have an important potential in wound dressing applications [11-13]. In literature it is emphasized that the chemistry of a polyurethane membrane has an important effect on biocompatibility [14]. In our previous study, triglyceride oil-modified polyurethane films for wound dressing applications were prepared from linseed oil-based hydroxyl containing material and hexamethylene diisocyanate (HMDI) and/or diphenylmethylene diisocyanate (MDI) [15]. It was found that the amount and type of diisocyanates affected the film and mechanical properties, and gas permeability of polymeric membranes. Notably, films were flexible and permitted flow of oxygen and carbon dioxide.

Linseed oil consists of various fatty acids such as oleic, linoleic, and linolenic acids, and all of them consist of different number of double bonds. Due to its high linoleic and linolenic acid content (approximately 70\% total), linseed oil has a tendency to oxidize with atmospheric oxygen. During the oxidation reaction, crosslink bonds between fatty acid chains form and the crosslink density increases, hence film formation is obtained. For this reason linseed oil is widely used in the formulation of polymers prepared as surface coating material. Some properties of the linseed oil-based polymer films, such as mechanical, thermal, viscoelastic, and surface coating properties, are directly related to the crosslink density which is proportional to the concentration of double bonds. Each linoleic acid chain contains two double bonds which is the optimum value in the formulation of an oil-based coating material. In this study, linoleic acid was used for the synthesis of polyurethane films to control the crosslink density. In the first step, hydroxyl containing component was synthesized by the esterification of linoleic acid and glycerol. In the second step, polyurethane was prepared using toluene 2,4-diisocyanate as the isocyanate component. We also aimed to eliminate catalyst in the polyurethane reaction and film preparation step in order to prevent possible negative effects on biocompatibility properties. For this purpose, polyurethane was synthesized without catalyst, films were prepared without crosslink-catalyst, and their properties were compared with those of the films prepared from catalyzed-reaction product with crosslink-catalyst.

\section{Experimental}

\subsection{Materials}

Toluene 2,4-diisocyanate (TDI) (Merck), linoleic acid (Fluka), glycerol anhydrous (Merck), and high purity xylene (Merck) were used without any further purification. Commercially purchased calcium octoate was used as catalyst for polymerization reaction and crosslink-catalyst. An Opsite ${ }^{\circledR}$ (Smith \& Nephew) brand commercial wound dressing was used as a comparison material.

\subsection{Polymer synthesis}

Polymer synthesis was achieved in two steps as described in the literature [9]: (1) preparation of hydroxyl containing component (HCC) from linoleic acid and glycerol, and (2) synthesis of polyurethane from HCC and TDI (Scheme 1).

\subsubsection{Preparation of hydroxyl containing material}

HCC was synthesized with a 2:1 mol ratio of linoleic acid and glycerol in the presence of xylene as a solvent. The reaction was carried out at $220^{\circ} \mathrm{C}$ with $p$-toluene sulfonic acid $(0.1 \%$ by weight with respect to the mixture of linoleic
Scheme 1 Polyurethane synthesis
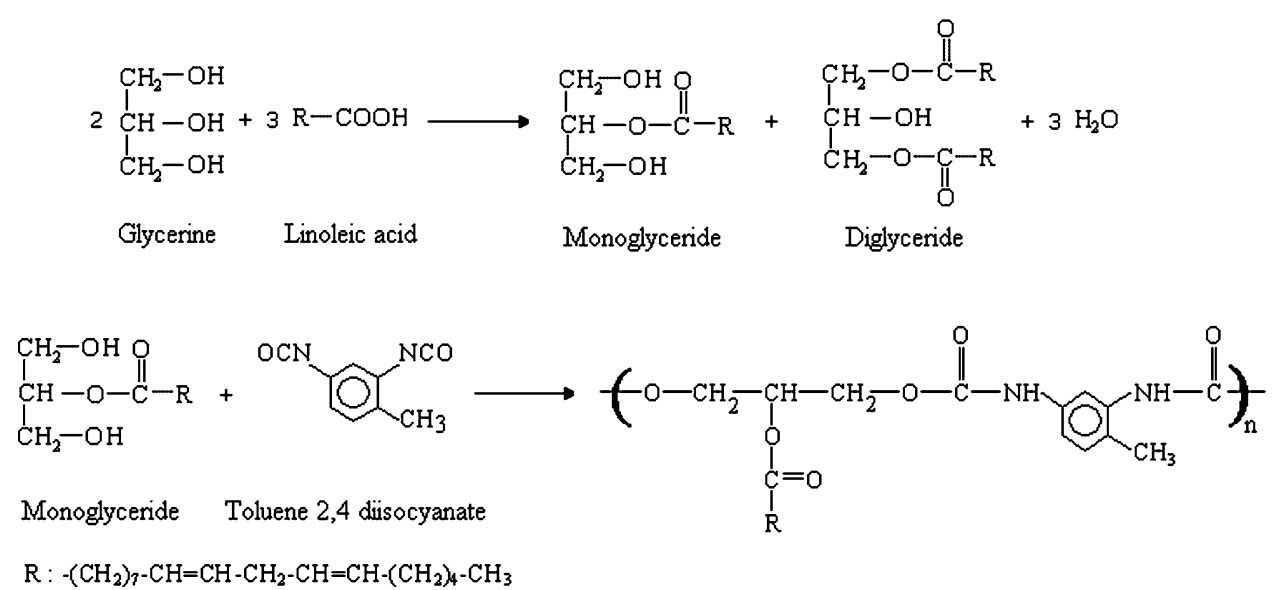
acid and glycerol) as a catalyst. Reaction was monitored by determination of acid value of the reaction mixture which was $120 \mathrm{mg} \mathrm{KOH} / \mathrm{g}$ at the beginning of the reaction. Acid value decreased during the reaction and attained about $3 \mathrm{mg} \mathrm{KOH} / \mathrm{g}$ at the end of the reaction. After removing the solvent, a sufficient amount of diethyl ether was added to dilute the mixture and the mixture is then washed with distilled water to remove the catalyst and free glycerol. The ethereal solution was then dried over $\mathrm{Na}_{2} \mathrm{SO}_{4}$, since the urethane formation reaction in the second step is sensitive to moisture, followed by solvent evaporation. The hydroxyl and acid values of HCC were $138.6 \mathrm{mg} \mathrm{KOH} / \mathrm{g}$ and $3.8 \mathrm{mg}$ $\mathrm{KOH} / \mathrm{g}$, respectively.

\subsubsection{Polyurethane synthesis}

Dried HCC and extra pure xylene were taken into the reaction flask and heated to $40-50^{\circ} \mathrm{C}$. An equivalent amount of TDI was added into the flask in a 30 min period. Calcium octoate was added to the reaction mixture in the amount of $0.02 \%$ (by weight) of HCC content for catalyzed-reaction. Then the reaction flask was heated to $90^{\circ} \mathrm{C}$ and reaction was carried out at this temperature under nitrogen atmosphere. Nitrogen atmosphere is necessary for both preventing oxidation of double-bonds on the acid chain and avoiding water since the reaction is sensitive to moisture. The reaction was monitored by Fourier transform infrared (FTIR) spectroscopy. The disappearance of the absorption peak at $2250 \mathrm{~cm}^{-1}$, assigned to the $\mathrm{N}=\mathrm{C}=\mathrm{O}$ group, was sought to confirm that all the diisocyanate were consumed in the reaction.

The polymers were coded as PU- $a$, where $a$ represents polyurethane preparation stage with (WC) or without (NC) catalyst.

\subsection{Preparation of polyurethane films}

Polyurethane films (100 $\mu \mathrm{m}$ thick) were prepared by casting evaporation technique. Films with controlled thickness were solution cast on a Teflon coated glass plate into a steel ring. Before transferring polymer solution into steel ring, it was stirred for $24 \mathrm{~h}$ with a magnetic stirrer. For preparing polymer films with crosslink-catalyst, calcium octoate was added ( $0.6 \mathrm{wt} . \%$ of the polymer) before transferring polymer solution into the steel ring. Cast films were kept at room temperature for $48 \mathrm{~h}$ and under vacuum at $45^{\circ} \mathrm{C}$ for $24 \mathrm{~h}$ for solvent removal. As the solvent was being removed from the film, oxidative polymerization of double bonds on fatty acid chain was also achieved and hence the polymer was cured. Dry film thickness was measured by dry film thickness gauge. For biocompatibility studies the films with a $20 \mu \mathrm{m}$ thickness were prepared using the same method.
The polymer films were designated using the abbreviation PU- $a-b$, where $a$ indicates the stage of polyurethane preparation with (WC) or without (NC) catalyst, and $b$ indicates the stage of film preparation with (c) or without crosslink-catalyst (nc).

\subsection{Characterization of polymers and polymer films}

FTIR spectroscopy analysis was carried out on a Perkin Elmer spectrometer by using the ATR mode. ${ }^{1} \mathrm{H}$ NMR spectra were obtained in $\mathrm{CDCl}_{3}$ using a $250 \mathrm{MHz}$ Bruker spectrometer and tetramethylsilane as the internal standard. Molecular weight of the polymers was determined by gel permeation chromatography (GPC) (Agilent 1100) equipped with a differential refractometer, at a flow rate of $1 \mathrm{ml} /$ min, using tetrahydrofuran as the solvent. Calibration of GPC was performed by using polystyrene standards. The morphological characterization of the polymer films was carried out by examining the surface of the membranes by scanning electron microscopy (SEM) (JEOL JSM-5410). SEM samples were prepared by breaking them in liquid nitrogen and then coating with gold using a sputter coater. The morphology of polymer films was determined by using wide angle X-ray scattering (WAXRD). X-Ray diffraction data were collected by X'PERT PRO X-ray diffractometer in the $2 \theta$ range $5-60^{\circ}$.

\subsection{Thermal, viscoelastic and mechanical properties}

The thermal gravimetric analysis (TGA) studies were carried out using a TA Q50 analyzer, by heating from room temperature to $600^{\circ} \mathrm{C}$, under nitrogen atmosphere at a heating rate of $20^{\circ} \mathrm{C} / \mathrm{min}$. The differential scanning calorimetry (DSC) measurements were carried out on a Perkin Elmer Diamond DSC between -100 and $200^{\circ} \mathrm{C}$ with a heating rate of $20^{\circ} \mathrm{C} / \mathrm{min}$.

The viscoelastic properties of polymer films were determined by Perkin Elmer Diamond dynamic mechanical analyzer (DMA) operating in tensile mode. The relaxation spectrum was scanned from -60 to $100^{\circ} \mathrm{C}$ with a frequency of $1 \mathrm{~Hz}$ and heating rate of $3^{\circ} \mathrm{C} / \mathrm{min}$.

Mechanical properties of polymer films were determined by a Shimadzu AGS-J Universal Tensile Tester at room temperature at an operating speed of $30 \mathrm{~mm} / \mathrm{min}$. For each film, several runs were carried out and the average value of these runs was used.

Tests for the samples were also performed on a Hysitron TriboIndenter ${ }^{\circledR}$, which is a high-resolution nanomechanical test instrument. Data for viscoelastic properties and mechanical properties were obtained by this instrument. The samples were mounted on small steel discs using superglue and held on the stage magnetically. Tests were 
carried out using a diamond indenter with Berkovich geometry for both dry state and water medium at $10 \mathrm{~Hz}$.

\subsection{Surface coating properties}

Films were prepared by dip coating method for determination of surface coating properties. For this purpose each polymer was diluted with xylene to $60 \mathrm{wt} . \%$ polymer content. Lead naphthenate $(0.5$ wt. $\%)$ and cobalt naphthenate $(0.05$ wt. $\%)$ were added as metal based on polymer content. The samples were allowed to stand $24 \mathrm{~h}$ before applying test surface. The surface coating properties such as set-to-touch drying time [16], water resistance [17] and flexibility [18] were determined according with the ASTM standards. In drying time determination, the polymer was coated on glass plate with a Bird film applicator $(60 \mu \mathrm{m}$ aperture) to give a wet film. The film was tested at intervals by touching lightly with a finger at points not less than $25 \mathrm{~mm}$ from the edge, and by touching a clean piece of glass. The set-to-touch time is the point at which the finger transfers no polymer to the clean glass. For water resistance tin plate was used as a substrate. After remaining in water for a period of time, coated plate was removed from the water, and was noted the time required for whitening, if any, to disappear for. Flexibility is related to elongation and was measured by the bending test. Polymer solution was applied on the tin plate. After drying the polymer, the plate was twisted upside down and cracks was observed. Acid resistance test was carried out at $25^{\circ} \mathrm{C}$ with $9 \%$ $\mathrm{H}_{2} \mathrm{SO}_{4}$ solution. After dropping acid solution onto polymer film it was covered with a watch glass to prevent liquid evaporation and the film damage was determined after $24 \mathrm{~h}$.

\subsection{Surface hydrophilicity, water absorption and biodegradation}

Static contact angle measurements were made using KSV CAM200 goniometer. For each measurement $5 \mu$ of distilled water was placed on the polyurethane surface. Water absorption properties were determined by immersing polymer films $(2 \times 1 \mathrm{~cm})$ into distilled water at $25^{\circ} \mathrm{C}$ until equilibrium was reached. Percent water absorption was determined gravimetrically by weighing the water saturated films after removing excess water on the film surfaces with a paper towel. Hydrolytic degradation was determined by immersing dry polymer films $(2 \times 1 \mathrm{~cm})$ in phosphate buffered saline (BPS) at $\mathrm{pH} 7.4$ and $37^{\circ} \mathrm{C}$. The test was continued for 7 weeks and 7 specimens were used for each polymer film. Every week one of the specimens were removed and weighed after drying in vacuum for 2 days. The weight loss was determined by using the measurements before and after BPS incubation.
2.8 Gas permeability

The gas permeabilities of the membranes were measured in a gas permeability system based on constant volume/variable pressure technique [19]. All measurements were carried out at $35^{\circ} \mathrm{C}$ and the permeation rates of $\mathrm{O}_{2}, \mathrm{~N}_{2}$, and $\mathrm{CO}_{2}$ gases were determined. Each membrane was tested three times and the average permeability value was reported. The reproducibility tests indicated a maximum measurement error of $\pm 5 \%$. Ideal selectivities were also determined by calculating the ratio of the pure gas permeabilities.

\subsection{Cytotoxicity of polyurethane films}

Polymer films were sterilized by ethylene oxide before cytotoxicity tests. Two methods were employed for determination of cytotoxicity; direct contact and MMT tests.

In the direct contact test, Murine fibroblast NIH 3T3 cell line (seeding density $1.5 \times 10^{4}$ per well) were precultured for $18 \mathrm{~h}$ in Dulbecco's modified essential medium supplemented with bovine serum (10\%) in 96-well plates and exposed for $24 \mathrm{~h}$ to the polymers placed in the center of each well. Commercially available wound dressing material (Opsite) was also tested for control whereas a growth medium, containing cells but no polymer, was tested for negative control. The morphological changes indicating cytotoxicity and cell growth characteristics were recorded using an NIKON-TMS microscope and attached Canon PC1049 camera.

In MTT test, after $24 \mathrm{~h}$ of cell culturing in the presence of individual polymer, the medium was removed and $100 \mu \mathrm{l}$ of growth medium with MTT $(5 \mathrm{mg} / \mathrm{ml}$ in PBS) was added to the cultures. Cells were incubated at $37^{\circ} \mathrm{C}$ in humidified atmosphere for $3 \mathrm{~h}$. Then the growth medium was removed, $100 \mu \mathrm{l}$ of lysis solution including $99.4 \%$ dimethyl sulfoxide (DMSO), $0.6 \%$ acetic acid, $10 \%$ sodium dodecylsulphate (SDS) was added to each well to dissolve purple crystals of formazan. Dissolved formazan of each well were transferred to another 96-well plate and the absorbance was measured in a spectrophotometer at a wavelength of $570 \mathrm{~nm}$. Reported values are the means of three replicates and are expressed as percentages of the control values.

\section{Results and discussion}

\subsection{Polymer synthesis and characterization}

$\mathrm{HCC}$ is an ester product of glycerol and linoleic acid, and hence the polymer chains include ester bonds besides urethane bonds. The structures of polymers were confirmed by FTIR and NMR measurements. 
Fig. 1 FTIR spectra of polyurethanes

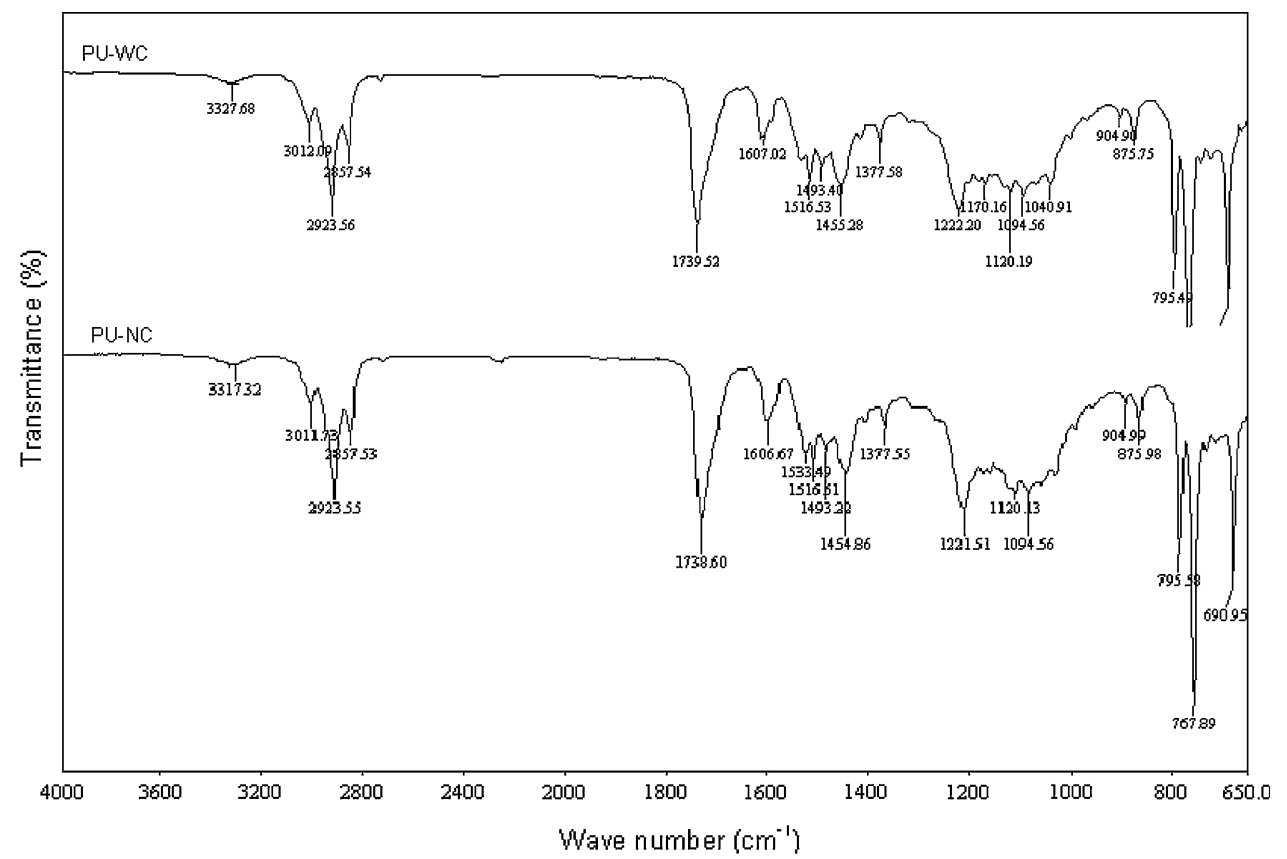

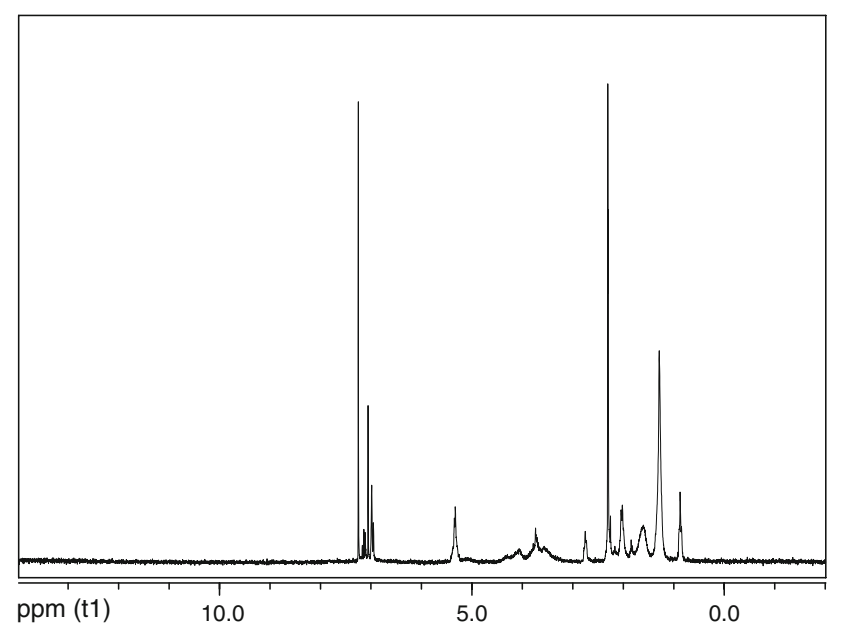

Fig. $2{ }^{1} \mathrm{H}$ NMR spectra of PU-WC

The FTIR spectra of polyurethanes prepared with (PUWC) and without (PU-NC) catalyst are presented in Fig. 1. A typical FTIR spectrum of both polyurethanes shows characteristic absorption band for $\mathrm{C}-\mathrm{N}-\mathrm{H}$ bending at $1533 \mathrm{~cm}^{-1}$. The peak around $1740 \mathrm{~cm}^{-1}$ is attributed to the carbonyl stretching of ester group. The peak at $1598 \mathrm{~cm}^{-1}$ has been assigned to the aromatic ring in polymer structure.

The ${ }^{1} \mathrm{H}$ NMR spectrum of PU-WC is shown in Fig. 2. The aromatic protons show peaks around 7.18-7.0 ppm and the peak at $2.26 \mathrm{ppm}$ is due to the methylene group attached to the aromatic ring originating from TDI [20]. The urethane $\mathrm{N}-\mathrm{H}$ proton in TDI-based polyurethane is expected to show peaks between 9.47-9.1 and 8.43$8.38 \mathrm{ppm}$ [21]. The peaks do not appear in the NMR
Table 1 Molecular weight and polydispersity of polyurethanes

\begin{tabular}{lll}
\hline Polyurethane & $\mathrm{M}_{\mathrm{w}}$ & Polydispersity \\
\hline PU-WC & 29000 & 7.3 \\
PU-NC & 27000 & 6.8 \\
\hline
\end{tabular}

spectra. The absence of these peaks may be due to the existence of hydrogen bonding between $\mathrm{C}=\mathrm{O}$ and $\mathrm{N}-\mathrm{H}$ groups in the polymer chain [22].

The molecular weights of the polymers are given in Table 1 . The value of weight average molecular weight $\left(\mathrm{M}_{\mathrm{w}}\right)$ for both polymers (PU-WC and PU-NC) is high enough and they are very close to each other. However, the value of polydispersity index is high for both polymers because of the structure of HCC. As presented above in Scheme 1, HCC was prepared by esterification of linoleic acid with glycerol. The reaction product is a mixture of monoglyceride and diglyceride having two and one hydroxyl group(s), respectively. Polymer chain is formed when the monoglyceride molecules react with diisocyanate molecules. Diglyceride molecules cause formation of short polymer chains. The reactivity of hydroxyl groups attached to $\alpha$ - and $\beta$-position of di- and monoglyceride molecules are different, however, it is not possible to select the hydroxyl group to react with isocyanate during the polymerization reaction. Thus, the composition of HCC is an important effect on the polydispersity of the final polymer.

During the preparation of polymer films, crosslinked structure is obtained due to oxidative polymerization with atmospheric oxygen [23]. The reaction mechanism is shown in Scheme 2. Although the reaction can be achieved 


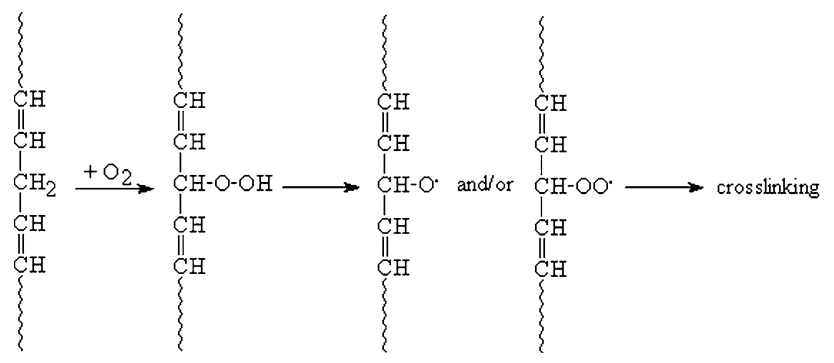

Scheme 2 Oxidative polymerization reaction

with or without crosslink-catalyst, the existence of crosslink-catalyst reduces the induction period and the quantity of oxygen necessary, increases oxygen absorption rate, and assists the formation and decomposition of peroxide which is the intermediate product in the crosslinking reaction [24]. The effect of crosslink-catalyst on some properties of polyurethane films prepared in this study is explained below.

$\mathrm{X}$-ray diffraction patterns of polyurethanes are given in Fig. 3. The strong broad peak around $2 \theta=20-25$ indicates a crystalline phase due to the presence of long aliphatic hydrocarbon chain of linoleic acid and hydrogen bonding in polyurethane. Polymer films prepared from uncatalyzedreaction product with (PU-NC-c) and without crosslinkcatalyst (PU-NC-nc) exhibit some sharp peaks around $2 \theta=30-35$. Since the uncatalyzed-reaction was continued

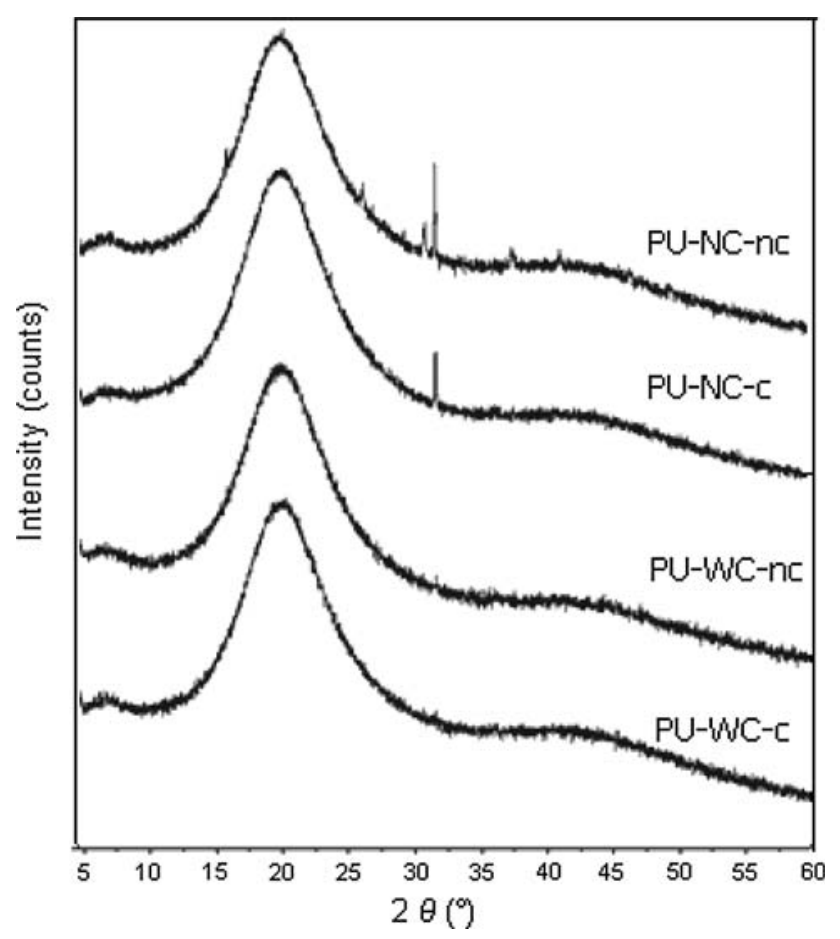

Fig. 3 WAXRD pattern of polyurethane films a long time in polyurethane synthesis stage, the crystalline regions may be introduced into the polymer structure.

\subsection{Thermal, viscoelastic and mechanical properties}

Figure 4 presents the TGA thermograms of polyurethanes prepared. All films show more than one decomposition stage. Although there is no significant difference, the films prepared without crosslink-catalyst (PU-NC-nc and PUWC-nc) have better thermal stability than the films prepared with crosslink-catalyst (PU-NC-c and PU-WC-c) between 215 and $375^{\circ} \mathrm{C}$. The crosslink-catalyst, calcium octoate, was also used as the catalyst in the preparation of PU-WC-nc in polymerization stage, but its amount was less than $0.02 \%$. Prasath et al. [21] and Jayakumar et al. [25] have already reported that the thermal stability of metal containing poly(urethane-ether)s decreases with increasing metal salt content. This behavior was explained with the fact that the metal catalyzes the first stage of decomposition and retards the further stages. Similar trend was observed for the films prepared in this study (Table 2). The decomposition temperature at low weight loss is higher for the polymer films prepared without crosslink-catalyst (PUNC-nc and PU-WC-nc). At high weight loss the decomposition temperature difference of films prepared with and without crosslink-catalyst is getting smaller.

One of the most important thermal data for an amorphous polymer is glass transition temperature $\left(\mathrm{T}_{\mathrm{g}}\right)$. It

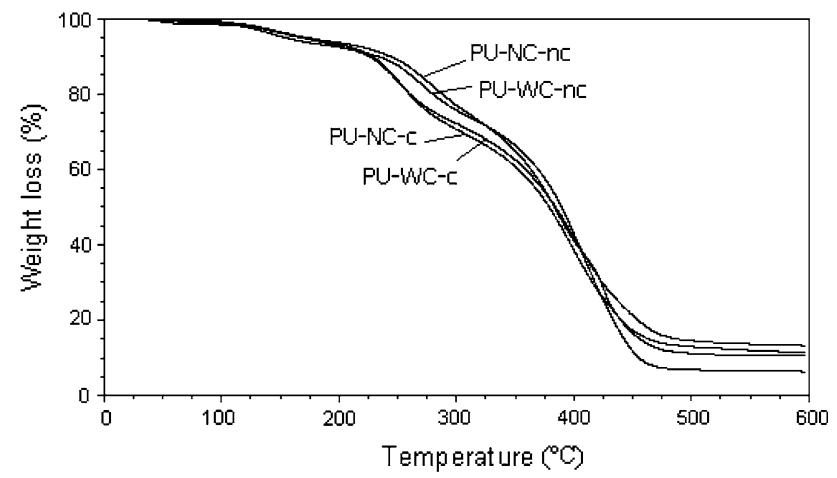

Fig. 4 TGA thermograms of polyurethane films

Table 2 Thermal properties and crosslinking density of the polyurethanes

\begin{tabular}{llllllll}
\hline Films & \multicolumn{3}{l}{$\begin{array}{l}\text { Temperature at weight } \\
\text { loss }\left({ }^{\circ} \mathrm{C}\right)\end{array}$} & & \multicolumn{2}{c}{$\mathrm{T}_{\mathrm{g}}\left({ }^{\circ} \mathrm{C}\right)$ by } \\
\cline { 2 - 3 } \cline { 7 - 8 } & $20 \%$ & $40 \%$ & $60 \%$ & & DSC & DMA & NanoDMA \\
\hline PU-NC-c & 260 & 352 & 398 & & 21 & 42 & - \\
PU-NC-nc & 287 & 363 & 402 & & 18 & 38 & 80 \\
PU-WC-c & 260 & 359 & 404 & & 19 & 42 & - \\
PU-WC-nc & 280 & 369 & 404 & & 10 & 32 & 60 \\
\hline
\end{tabular}



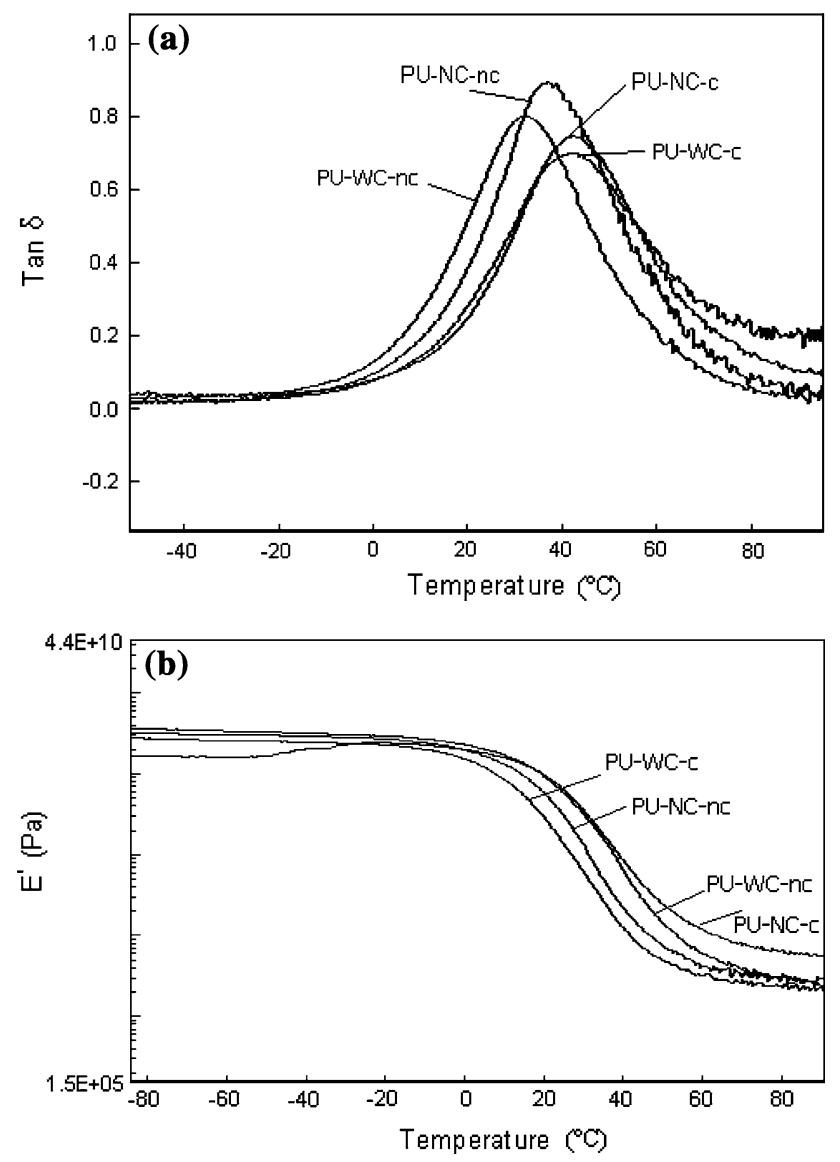

Fig. 5 Viscoelastic properties of polyurethane films. (a) Tan $\delta$ and (b) storage modules

indicates a critical temperature for the polymer that limits its technical application (at temperatures higher than glass transition). When the value of $\mathrm{T}_{\mathrm{g}}$ determined by DMA identified from the peak of the $\tan \delta$ curve (Fig. 5a) are compared to that determined from DSC method, the latter gave lower values of $\mathrm{T}_{\mathrm{g}}$, while the trend was the same, films prepared with crosslink-catalyst have higher $\mathrm{T}_{\mathrm{g}}$ (Table 2). One of the parameters affecting the $\mathrm{T}_{\mathrm{g}}$ is the crosslink density. The $\mathrm{T}_{\mathrm{g}}$ increases with increasing degree of crosslinking [26]. In this study, crosslinked polyurethane films were obtained during the curing process where the double bonds on linoleic acid chain reacted with atmospheric oxygen to form crosslinks between the double bonds. As expected, using crosslink-catalyst caused an increase in $\mathrm{T}_{\mathrm{g}}$ (Table 2) because of increasing crosslink density. This result was confirmed by DMA (Fig. 5). The storage modulus is related to the degree of crosslink density $\left(v_{\mathrm{e}}\right)$ calculated using

$v_{e}=\mathrm{E}^{\prime} / 3 \mathrm{RT}$

where $E^{\prime}$ is the storage modulus value obtained in the rubbery plateau, $\mathrm{T}$ is temperature in degrees $\mathrm{K}$ corresponding to the storage modules value, and $\mathrm{R}$ is the gas constant [27]. It is clear that crosslink density is directly proportional to storage modulus. Since the value of E' is the highest in the rubbery plateau for PU-NC-c, it has slightly higher crosslink density compared to the other polymer films (Fig. 5b). This film was prepared in the presence of crosslink-catalyst from polyurethane synthesized without catalyst. On the other hand, PU-WC-c was also prepared in the presence of crosslink-catalyst, but its crosslink density is smaller than that of PU-NC-c. This result can be explained with the different reaction conditions during polymer synthesis. Although both reactions were carried out at the same temperature, using catalyst decreased the reaction time from 15 to $3.5 \mathrm{~h}$. Long reaction time may allow the formation of the crystalline phase, as confirmed by X-ray results.

The two samples prepared without crosslink-catalyst (PU-NC-nc and PU-WC-nc) have higher $\tan \delta$ peak than the samples prepared without crosslink-catalyst (PU-NC-c and PU-WC-c). The highest tan $\delta$ peak was obtained for PU-NC-nc sample. This result shows that PU-NC-nc film is more elastic than others.

In order to measure the viscoelastic properties of microstructural features in polymer and mapping of mechanical properties in tissues with complex microstructures, the samples prepared without crosslink-catalyst (PU-NC-nc and PU-WC-nc) were fully characterized by nanoDMA. Although, for indenting soft polymers a spherical tip is proposed to avoid damaging the sample and minimize plastic deformation [28], a Berkovich tip was used for indenting the samples prepared because of the non-opportunity to select the tip. Nevertheless, the results were confirmed by the DMA results. The glass transition temperature was found to be $60^{\circ} \mathrm{C}$ for PU-WC-nc and $80^{\circ} \mathrm{C}$ for PU-NC-nc (Fig. 6). Although the trend is the same, the values of $\mathrm{T}_{\mathrm{g}}$ for each film are not the same obtained from both DMA and nanoDMA. It is well known that different $T_{g}$ values are determined for the

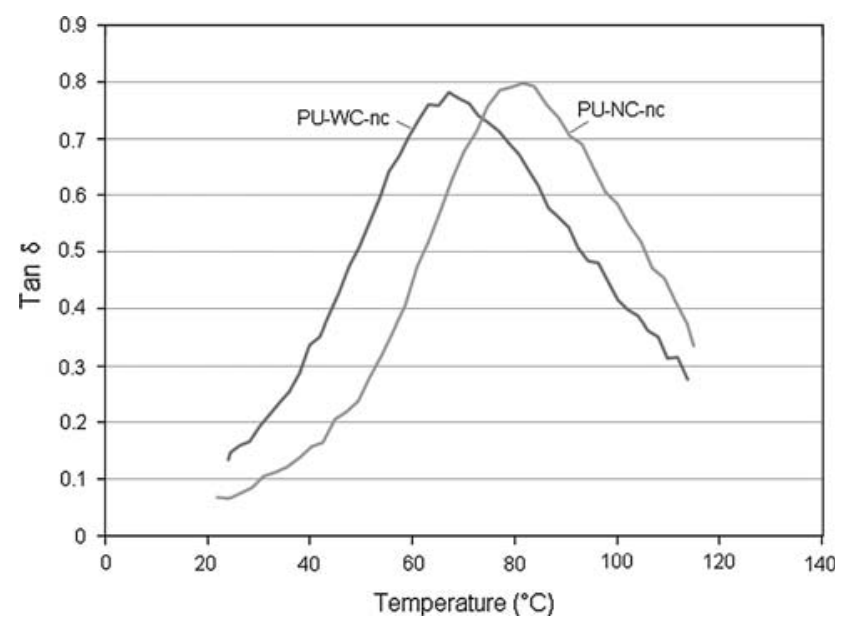

Fig. 6 Tan $\delta$ peaks 
same polymer depending on the test method used (such as DMA and DSC methods) and the test conditions. Although the methods are the same for nanoDMA and DMA, the test conditions are not the same. The frequency is 1 and $10 \mathrm{~Hz}$ for DMA and nanoDMA, respectively. For this reason the data obtained from two devices can not be compared.

When applied to the body the artificial skin is exposed to the body liquids; as a simple and first approach the samples were immersed in water. Further studies should use liquids comparable to the body liquids because the dipole momentum of the liquid can have significant impact on the result. Figure 7 shows the instantaneous effect of the water on the $\tan \delta$ value of the samples. With the indenter the sample was tested from the same side that is immersed in water. Studies from the opposite side would allow determining how fast the water diffuses into the sample.

Mechanical properties of polymer films are shown in Table 3. The increase in the tensile strength and decrease in the elongation are due to the relatively high crosslink density in the polymer structure. The films prepared in the presence of crosslink-catalyst increased the crosslink density and thus increased the tensile strength while decreasing elongation at break.

The hardness and reduced elastic modules were calculated from the loading/unloading curves of nanoindentation

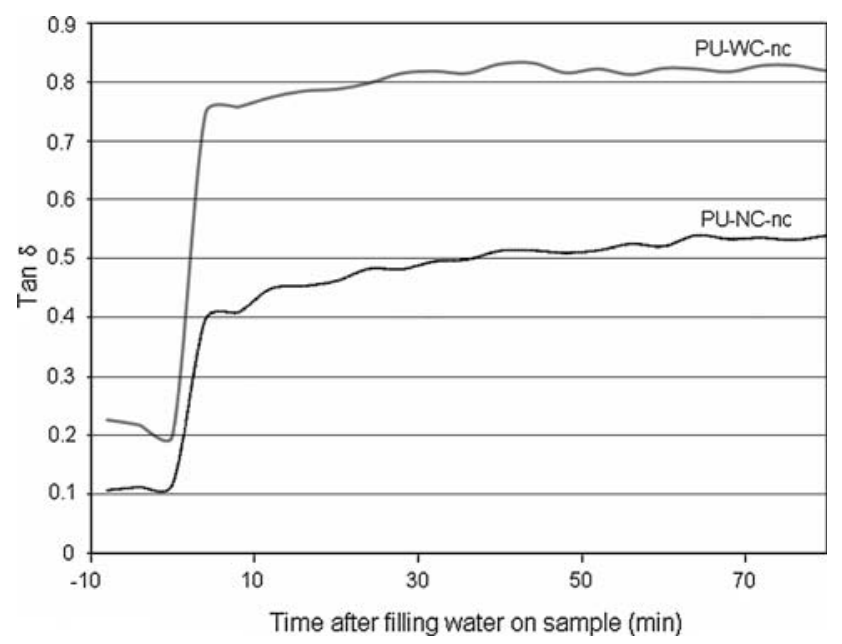

Fig. 7 Tan $\delta$ curves in water medium

Table 3 Mechanical properties of the polyurethanes

\begin{tabular}{lll}
\hline Films & $\begin{array}{l}\text { Tensile } \\
\text { strength }(\mathrm{MPa})\end{array}$ & $\begin{array}{l}\text { Elongation } \\
\text { at break }(\%)\end{array}$ \\
\hline PU-NC-c & 5.4 & 25.2 \\
PU-NC-nc & 2.2 & 55.5 \\
PU-WC-c & 5.6 & 28.9 \\
PU-WC-nc & 2.2 & 64.1 \\
\hline
\end{tabular}

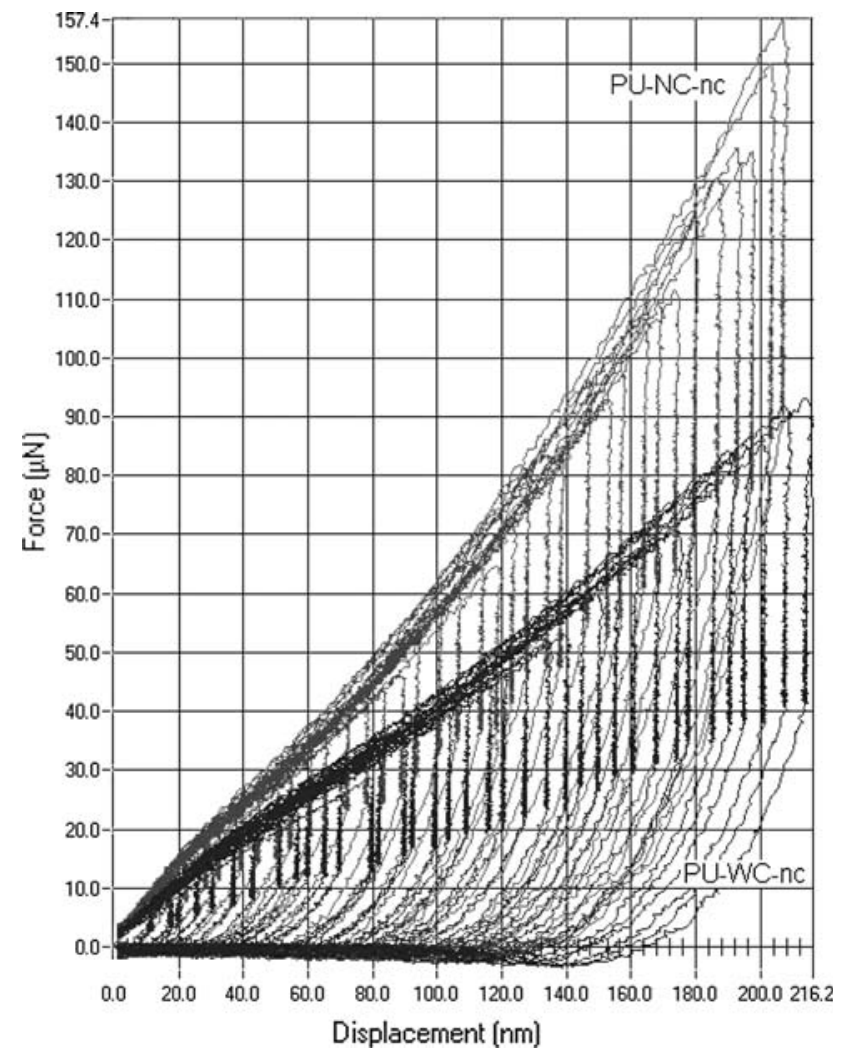

Fig. 8 Loading/unloading curves

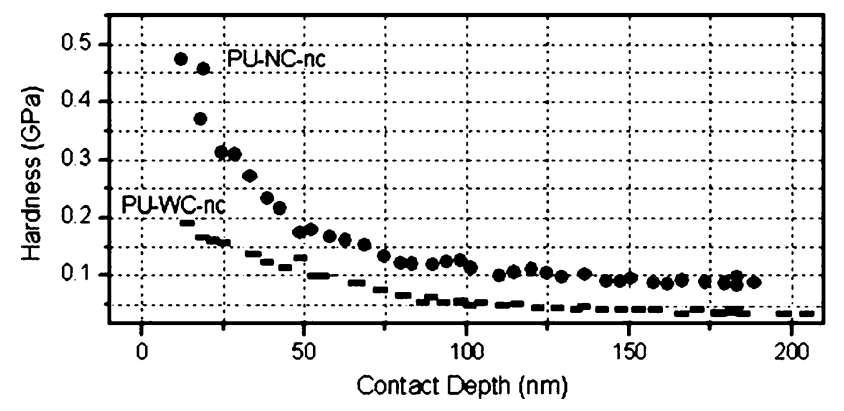

Fig. 9 Hardness curves

(Fig. 8) for each sample. The applied force for indentation was higher for PU-NC-nc film than that of PU-WC-nc for the same displacement. This result shows that PU-NC-nc film is harder than PU-WC-nc film (Fig. 9). Reduced modules $\left(\mathrm{E}_{\mathrm{r}}\right)$ is related to the modules of elasticity. PUNC-nc film is more elastic than PU-WC-nc because of the higher $\mathrm{E}_{\mathrm{r}}$ (Fig. 10). This result was confirmed by DMA as the highest $\tan \delta$ peak for PU-NC-nc film.

\subsection{Surface coating properties}

Surface coating properties were investigated for the films prepared in the presence of crosslink-catalyst (PU-NC-c and PU-WC-c). The drying time which indicates the rate of 


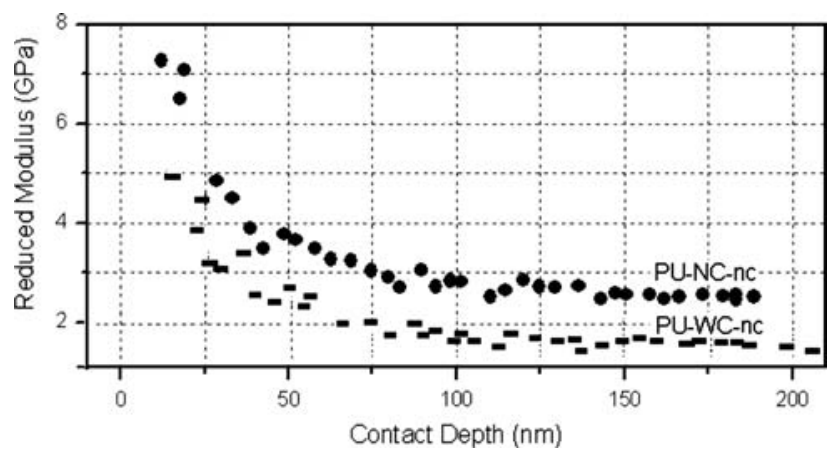

Fig. 10 Reduced modules curves

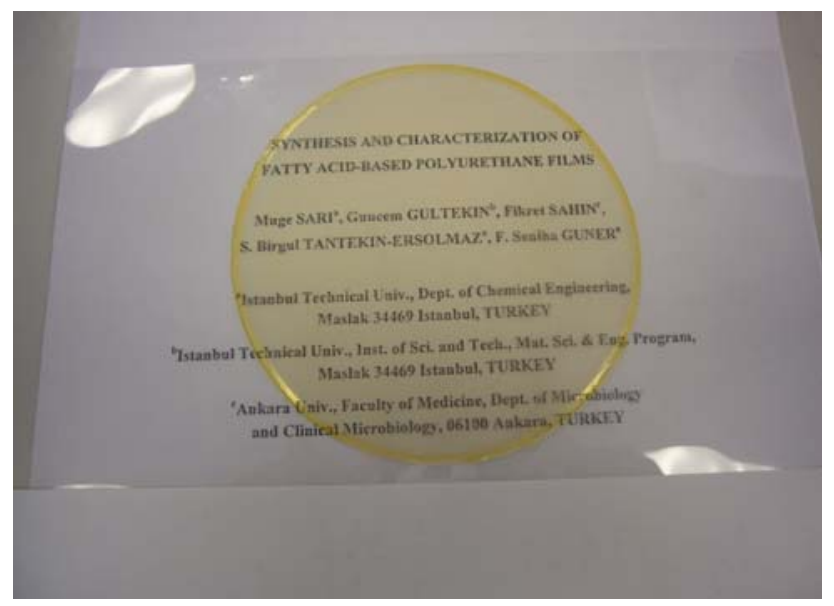

Fig. 11 Transparent dry polyurethane film

film formation during casting step was determined as 5 and 7 min for PU-WC-c and PU-NC-c, respectively. Since Opsite $^{\circledR}$ is sold in the film form, it was not possible to determine its drying time.

Water resistance was superior for all films, since the time required for whitening to disappears was short, 26 and 30 min for PU-NC-c and PU-WC-c, respectively. On the other hand, water did not influence the commercial product Opsite ${ }^{\circledR}$ under test conditions. Both of the films and the commercial product were very flexible, because the cylinder with lowest diameter caused no crack on the film in the bending tests. Both films had high acid resistance and were yellow transparent color (Fig. 11).

\subsection{Surface hydrophilicity, water absorption and biodegradation}

Contact angle itself is not an indicator of biocompatibility of a material, but some useful information may be obtained for blood- or tissue-compatibility. The optimal water contact angle of a material for protein adsorption and cell
Table 4 Contact angel, water adsorption and biodegradation (after 7 weeks)

\begin{tabular}{lclc}
\hline Films & $\begin{array}{l}\text { Contact } \\
\text { angle }\left({ }^{\circ}\right)\end{array}$ & $\begin{array}{l}\text { Water } \\
\text { absorption }(\%)\end{array}$ & $\begin{array}{l}\text { Weight loss } \\
\text { in BPS }(\%)\end{array}$ \\
\hline PU-NC-c & 86 & 0.33 & 7.9 \\
PU-NC-nc & 50 & 9.87 & 15.6 \\
PU-WC-c & 72 & 1.13 & 6.4 \\
PU-WC-nc & 62 & 9.08 & 12.1 \\
Opsite & 110 & 0.8 & 3.6 \\
\hline
\end{tabular}

adhesion was suggested to be in the range of $40-65^{\circ}$ by Lee et al. [29].

The static water contact angle for the polyurethanes prepared is reported in Table 4. All films can be considered hydrophilic because their contact angles are smaller than $90^{\circ}$. The contact angles of the films prepared without crosslink-catalyst (PU-NC-nc and PU-WC-nc) are smaller than that of the films prepared with crosslink-catalyst, and they have optimal water contact angle for cell adhesion and protein adsorption. The contact angle for Opsite ${ }^{\circledR}$ was measured as $110^{\circ}$.

Table 4 also presents the water absorption capacity and the amount of hydrolytic degradation for all films prepared in this study. Since the films prepared without crosslinkcatalyst is more hydrophilic, they absorbed more water and lost more weight in PBS than the films prepared with crosslink-catalyst for the same experimental period. The increased hydrophilicity caused an increase in water absorption capacity and hydrolytic degradation. All films prepared are more biodegradable than the commercial product.

\subsection{Gas permeability}

The $\mathrm{O}_{2}, \mathrm{~N}_{2}$, and $\mathrm{CO}_{2}$ permeability coefficients of the polyurethane films under study here and the commercial product Opsite ${ }^{\circledR}$ are presented in Table 5. The fatty acid based polyurethane films all exhibited $\mathrm{O}_{2}, \mathrm{~N}_{2}$, and $\mathrm{CO}_{2}$ permeabilities considerably lower than the commercial product. The highest permeability values were generally obtained for the films prepared without crosslink catalyst

Table 5 Permeabilities and selectivities of the polyurethane films

\begin{tabular}{lllllll}
\hline Membrane & $\begin{array}{l}\mathrm{O}_{2} \\
\text { (Barrer) }\end{array}$ & $\begin{array}{l}\mathrm{N}_{2} \\
\text { (Barrer) }\end{array}$ & $\begin{array}{l}\mathrm{CO}_{2} \\
\text { (Barrer) }\end{array}$ & $\alpha_{\mathrm{O}_{2} / \mathrm{N}_{2}}$ & $\alpha_{\mathrm{CO}_{2} / \mathrm{N}_{2}}$ & $\alpha_{\mathrm{CO}_{2} / \mathrm{O}_{2}}$ \\
\hline PU-NC-c & 0.28 & 0.13 & 1.10 & 2.15 & 8.46 & 3.93 \\
PU-NC-nc & 0.53 & 0.43 & 2.14 & 1.23 & 4.98 & 4.04 \\
PU-WC-c & 0.30 & 0.08 & 1.43 & 3.75 & 17.88 & 4.77 \\
PU-WC-nc & 0.67 & 0.38 & 1.35 & 1.76 & 3.55 & 2.01 \\
Opsite & 9.9 & 2.97 & 75.9 & 3.3 & 25.25 & 7.6 \\
\hline
\end{tabular}


(PU-NC-nc, PU-WC-nc). This result is in agreement with the $T_{g}$ data where the films prepared without crosslink catalyst showed lower $T_{g}$ values than the films prepared with crosslink catalyst indicating lower free volume for the films without crosslink catalyst. As indicated before, the use of crosslink-catalyst increases the amount of peroxide groups and hence the crosslink density. The only exception to this observation is the $\mathrm{CO}_{2}$ permeability of PU-WC-nc which is slightly lower than PU-WC-c. However, this difference is approximately within the range of experimental error and is believed to have no physical significance.

The PU-NC-nc had slightly higher $\mathrm{N}_{2}$ and $\mathrm{CO}_{2}$ permeability coefficients than PU-WC-nc whereas PU-WC-nc had slightly higher $\mathrm{O}_{2}$ permeability. The $\mathrm{CO}_{2} / \mathrm{N}_{2}$ selectivities of the films also were lower than those of Opsite ${ }^{\circledR}$. The $\mathrm{O}_{2} / \mathrm{N}_{2}$ and $\mathrm{CO}_{2} / \mathrm{O}_{2}$ selectivities were of the same order of magnitude as those of the commercial product. The films prepared with crosslink catalyst gave higher $\mathrm{O}_{2} / \mathrm{N}_{2}$ selectivities than the films prepared without crosslinked catalyst. The films with higher permeabilities also gave lower selectivities as expected due to the permeability/selectivity trade off behavior of polymer films.

\subsection{Cytotoxicity}

The percentage of the cell proliferation on the polyurethane films after $24 \mathrm{~h}$ of incubation is given in Fig. 12. The cell proliferation on PU-NC-c and PU-NC-nc films is relatively close to the control sample and the commercial product. It should be noted that the film prepared from uncatalyzed reaction product and without crosslink-catalyst (PU-NCnc) gave the best result. Removing the catalyst from the polymer synthesis stage has a major advantage for cytotoxicity. The non-cytotoxicity of this film was also supported by the phase contrast micrographs (Fig. 13). PUNC-nc has the same morphology and cell growth characteristic as the control and the commercial sample Opsite ${ }^{\circledR}$. The studies about cytotoxicity determined by in vivo assay in using the contact test of irritability in rats are still in progress.

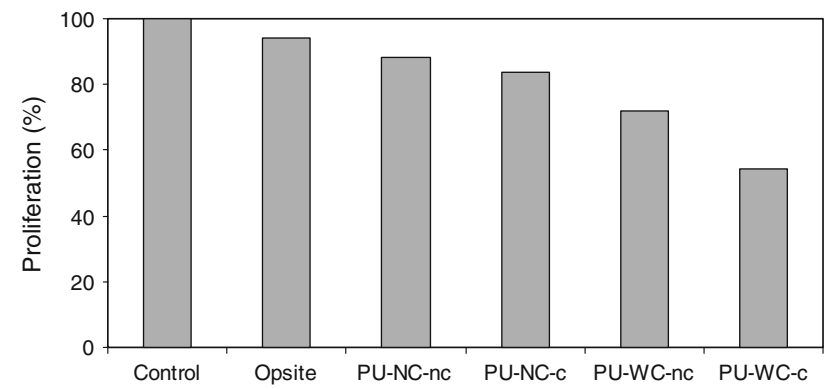

Fig. 12 The cell proliferation on the surface of polymer films
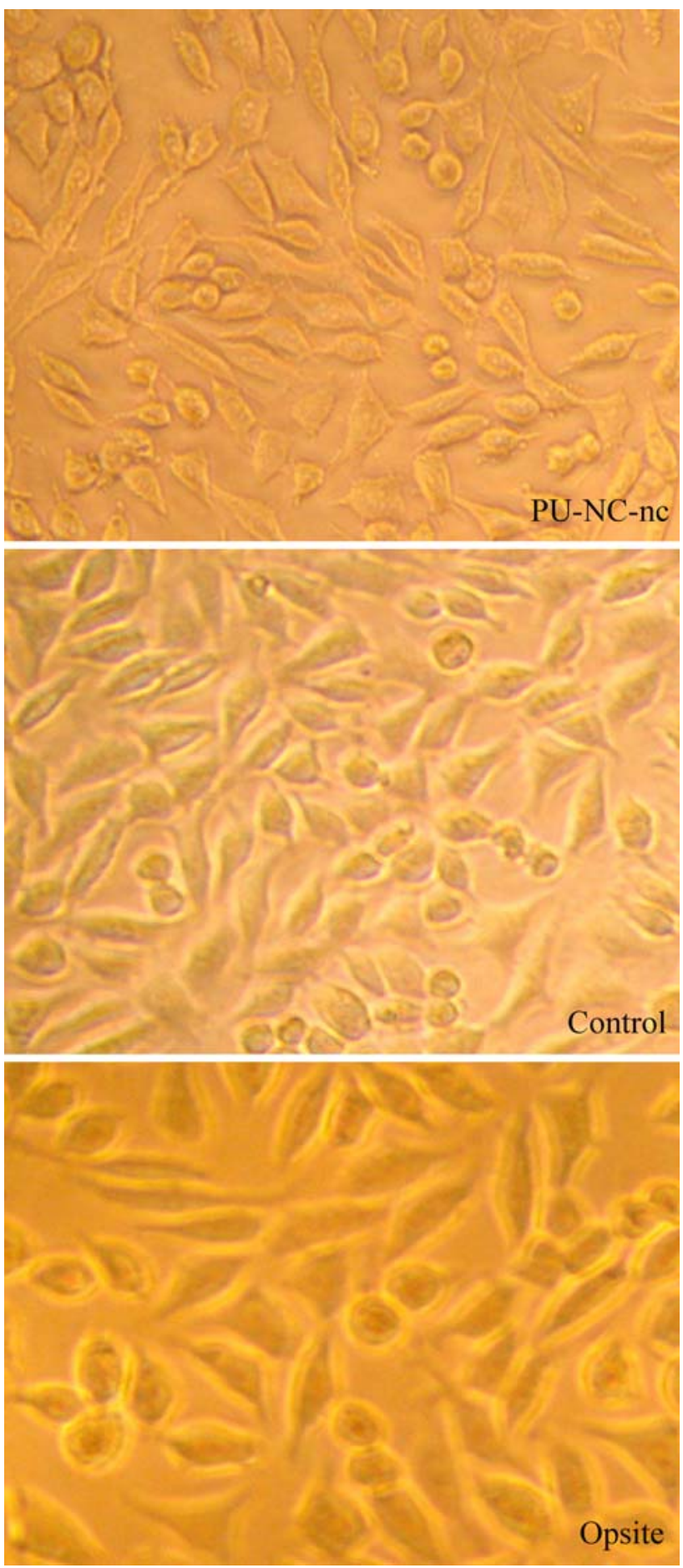

Fig. 13 Phase contrast micrographs

\section{Conclusion}

The linoleic acid-based polyurethane film were successfully prepared in the absence of any reaction and crosslinking catalyst (PU-NC-nc) and characterized for use as wound dressing material. Additionally, linoleic acid-based polymer 
films were prepared from uncatalyzed reaction product with crosslinking catalyst (PU-NC-c), catalyzed reaction product with (PU-WC-c) and without (PU-WC-nc) crosslinking catalyst. All films were transparent and flexible. The thermal, viscoelastic, mechanical, and surface coating properties of all polyurethane films were fairly good and appropriate use as wound dressing material.

From the biomedical point of view, the film prepared from uncatalyzed reaction product without crosslink-catalyst (PU-NC-nc) showed the highest water absorption and biodegradation, and the best cytotoxicity. $\mathrm{O}_{2}$ and $\mathrm{CO}_{2}$ permeabilities of this film were found higher. The film is hydrophilic and its contact angle was determined to be $50^{\circ}$. The film prepared from uncatalyzed reaction product with crosslink-catalyst (PU-NC-c) was also non-toxic, but water absorption, biodegradation, $\mathrm{O}_{2}$ and $\mathrm{CO}_{2}$ permeabilities were found lower than the PU-NC-nc. On the other hand, its $\mathrm{O}_{2} / \mathrm{N}_{2}$ selectivity was higher.

The novelty of this study is to use linoleic acid which is the main composition of some triglyceride oils, in the preparation of wound dressing. The obtained results will be helpful to the researchers with an interest in applications of fatty acids and renewable sources in biomedical applications.

Acknowledgments This research is partially funded by the State Planning Organization (DPT) of Turkey. We are grateful to Prof. Dr. Naciye Talinli for discussion of NMR and FTIR results.

Open Access This article is distributed under the terms of the Creative Commons Attribution Noncommercial License which permits any noncommercial use, distribution, and reproduction in any medium, provided the original author(s) and source are credited.

\section{References}

1. F.S. Guner, Y. Yagci, A.T. Erciyes, Prog. Polym. Sci. 31, 633670 (2006). doi:10.1016/j.progpolymsci.2006.07.001

2. D. Swern (ed.), Bailey's Industrial Oil and Fat Products, vol. 1, 6th edn. (John Wiley and Sons, New Jersey, 2005), pp. 1-9

3. T.T. Reddy, A. Kano, A. Maruyama, M. Hadano, A. Takahara, Biomacromolecules 9(4), 1313-1321 (2008)

4. M. Rogulska, A. Kultys, W. Podkoscielny, Eur. Polym. J. 43, 1402-1414 (2007). doi:10.1016/j.eurpolymj.2007.01.014

5. M. Rogulska, W. Podkoscielny, A. Kultys, Eur. Polym. J. 42, 1786-1797 (2006). doi:10.1016/j.eurpolymj.2006.02.014

6. A.T. Erciyes, F.S. Erkal, O.S. Kabasakal, J. Coat. Technol. 64(815), 61-64 (1992)
7. F.S. Güner, M. Baranak, S. Soytaş, A.T. Erciyes, Prog. Org. Coat. 50, 172-178 (2004). doi:10.1016/j.porgcoat.2003.12.004

8. O. Yılmaz, M. Baranak, F.S. Güner, A.T. Erciyes, J. Appl. Polym. Sci. 98, 1032-1035 (2005). doi:10.1002/app.22223

9. F.S. Guner, A. Gumusel, S. Calica, A.T. Erciyes, J. Coat. Technol. 74(929), 55-59 (2002). doi:10.1007/BF02698369

10. J.P. Santerre, K. Woodhouse, G. Laroche, R.S. Labow, Biomaterials 26, 7457-7470 (2005). doi:10.1016/j.biomaterials.2005. 05.079

11. N.M.K. Lamba, K.A. Woodhouse, S.L. Cooper, Polyurethanes in Biomedical Applications (CRC, Boca Raton, 1998), p. 38

12. M. Pavlova, M. Draganova, Biomaterials 14(13), 1024-1029 (1993). doi:10.1016/0142-9612(93)90196-9

13. K. Ulubayram, N. Hasırc1, Polymer (Guildf) 33, 2084-2088 (1992). doi:10.1016/0032-3861(92)90874-V

14. M.C. Belanger, Y. Marois, R. Roy, Y. Mehri, E. Wagner, Z. Zhang et al., Artif. Organs 24, 879-888 (2000)

15. M.U. Ozkaynak, C. Atalay Oral, S.B. Tantekin Ersolmaz, F.S. Guner, Macromol. Symp. 228, 177-184 (2005). doi:10.1002/ masy.200551016

16. ASTM D 1640-83, Test for drying, curing or film formation of organic coatings at room temperature, Annual Book of American Society for Testing and Material Standards, vol. 6 (Philadelphia, PA, 1991), pp. 242-245

17. ASTM D 1647-89, Resistance of dried film to vanishes, water and alkalie, Annual Book of American Society for Testing and Material Standards, vol. 6 (Philadelphia, PA, 1994), pp. 268-269

18. ASTM D522-93a, Standard test methods for mandrel bend test of attached organic coatings, Annual Book of American Society for Testing and Material Standards, vol. 6 (Philadelphia, PA, 2001)

19. M. Mulder, Basic Principles of Membrane Technology (Kluwer Academic Publishers, Dordrecht, 1991)

20. H. Sadtler, The Sadtler Handbook of Proton NMR Spectra (Heyden and Son Ltd., Philadelphia, 1978), pp. 1223-1225

21. R.A. Prasath, S. Nanjundan, T. Pakula, M. Klapper, Eur. Polym. J. 40, 1767-1778 (2004). doi:10.1016/j.eurpolymj.2004.03.007

22. P.A. Mirau, A Practical Guide to Understanding the NMR of Polymers (John Wiley and Sons, New Jersey, 2004), p. 24

23. K.F. Lin, Paints, varnishes and related products, in Bailey's Industrial Oil and Fat Products, ed. by F. Shahidi (John Wiley and Sons, New Jersey, 2005), pp. 307-318

24. P. Deligny, N. Tuck, Alkyd and Polyesters, in Resin for Surface Coatings, vol. 2, ed. by P.K.T. Oldring (John Wiley and Sons, New York, 2000), p. 38

25. R. Jayakumar, Y.S. Lee, M. Rajkumar, S. Nanjundan, J. Appl. Polym. Sci. 91, 288-295 (2003). doi:10.1002/app.13222

26. J.R. Fried, Polymer Science and Technology, 2nd edn. (Prentice Hall PTR, 2003), p. 183

27. L.W. Hill, Dynamic mechanical and tensile properties, in Paint and Coating Testing Manual 14th edition of the Gardner-Sward handbook, ed. by J.V. Koleske (ASTM Manual Series: MNL 17, Ann Arbor, MI, 1995), p. 541

28. D.M. Ebenstein, L.A. Pruitt, Nanoindentation of biological materials. Nanotoday 1(3), 26-33 (2006)

29. J.H. Lee, H.B. Lee, J. Biomater. Sci. Polym. Ed. 4, 467-481 (1993) 